The 100 who had a successful "take"(75\%) donated 215 units ( $82 \%$ plasma and $22 \%$ blood).

A detailed informative campaign must be conducted among healthcare personnel. Volunteer qualified blood and plasma donors should be considered as a reliable alternative for special campaigns.

Keywords: adverse events; antibodies; immunizations, mass; immunoglobulin: plasma; plasmapheresis; production; smallpox; vaccination; volunteers

Prebosp Disast Med 2003:18:s(1)s35.

\section{Five Decades of Voluntary Blood-Donation Service}

Srecko Zajc

Secretary General of Slovenian Red Cross

This year, we celebrate five decades of voluntary blooddonation service on the territory of Republic of Slovenia. Actually, this long tradition has its roots in the Partisans' improvisations during the Second World War. During the period of transition, many of the traditional issues were under question, e.g, where to go and how to find the proper answers on a new challenges? Even during such a turbulent time, everyone involved in the blood-donation process was responsible enough so that today, the Republic of Slovenia is self-sufficient with an adequate and safe supply of blood and its products.

Nowadays, we are faced with reorganization of the transfusion system so that the Slovenian Red Cross, as a major motivator and organizer of volunteers, must work more closely with experts to find a "new" volunteer. Such a volunteer probably works in an office, he (mostly not she) is well-educated, is able to use a personal computer (PC), and can be accessed using a mobile telephone. But, he has no time and is master of his free time. How can this new type of volunteer be motivated?

Another difficult task is to be prepared for all kinds of disaster, and to respond immediately to every request for an extra quantity of a fresh and necessary blood. It means more work not only for volunteers in the branches of Slovenian Red Cross, but also more well-coordinated work between medicals, Red Cross professionals, volunteers, and the Government including their plans and expenses.

Keywords: blood; blood products; coordination; donation; motivation; Red Cross; Slovenia; supplies; transfusion; volunteers Prehosp Disast Med 2003;18(s1)s35.

\section{Hemopure ${ }^{\otimes}$ (HBOC-201) Compared With Blood (PRBC) for Two-Unit Infusion in Orthopedic Surgery Following Trauma \\ Douglas M. Hansell, $M D$ \\ Biopure Corporation, Cambridge, Massachusetts, USA}

Introduction: Approximately 250,000 trauma patients require blood (PRBC) transfusions in the U.S. each year; 50,000 receive two units of PRBC or less. ${ }^{1}$ It was hypothesized that such two-unit PRBC exposure could safely be avoided by the alternative use of a hemoglobin-based $\mathrm{O}_{2}$ carrier (HBOC).

Methods: The physiological changes and adverse events (AEs) within 24 hours of a two-unit $\mathrm{HBOC}$ infusion of Hemopure $(n=34)$ were compared with two units of
PRBC $(\mathrm{n}=28)$ in 62 patients who had sustained blunt trauma and who underwent orthopedic surgery.

Results: $14 / 28$ (50\%) PRBC and 25/34 (74\%) HBOC201 patients had mean values of $1.04(\mathrm{CL}=0.54-1.53)$ and $1.44(\mathrm{CL}=0.96-1.92) \mathrm{AEs}$ as defined by the U.S. Food and Drug Administration (FDA), respectively. Five HBOC-201 and four PRBC patients had cardiovascular AEs; seven HBOC-201 and four PRBC patients had gastrointestinal AEs; and three HBOC-201 and one PRBC patient had pyrexia. Other AEs were numerically and systematically no different. Serious AEs with HBOC-201 included one vascular (HTN) and one respiratory failure. Mean and $95 \%$ confidence limits (CL) of serial hemoglobin concentrations $[\mathrm{Hb}]$ and systolic blood pressures (SBP) are shown in Table.

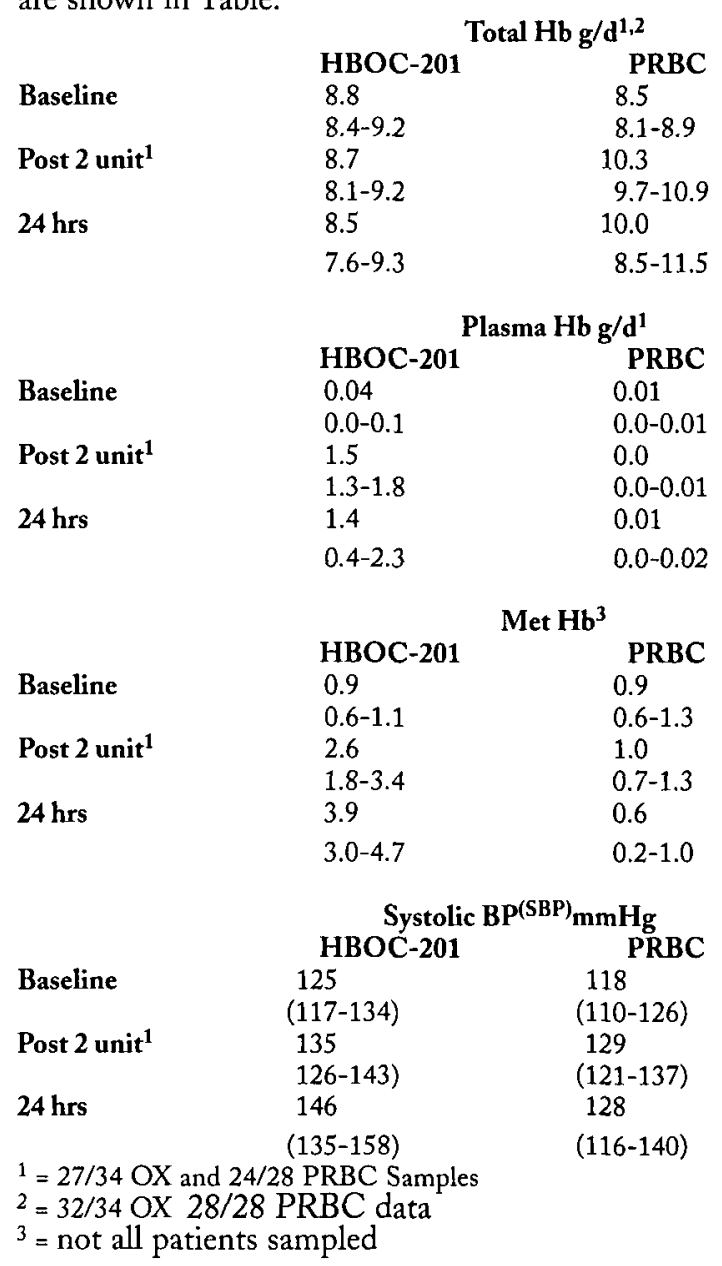

Discussion: Pyrexia, mild HTN, and gastrointestinal symptoms following HBOC are well recognized. ${ }^{2}$ A twounit HBOC-201 infusion ( $60 \mathrm{~g} / \mathrm{dL} \mathrm{Hb}$ ) would be expected to elevate plasma $\mathrm{Hb}$ by approximately $1.5-1.7 \mathrm{~g} / \mathrm{dL}$. The low plasma $\mathrm{Hb}$ in these blunt trauma patients may reflect either the oncotic effects or pharmacokinetics of $\mathrm{HBOC}-201$ or an interstitial leakage of plasma $\mathrm{Hb}$. Use of HBOC-201 could save 100,000 PRBC and avoid blood exposure by $20-25 \%$ or more nationwide.

Conclusion: Infusion of two units of HBOC-201 has the advantage of similar overall $A E s$ to the infusion of PRBC and of avoiding allogenic blood exposure. And the use of 